\title{
Projecting population growth as a dynamic measure of regional urban warming
}

Chenghao Wang, Zhi-Hua Wang*

School of Sustainable Engineering and the Built Environment, Arizona State University, Tempe, AZ 85287, USA

*Corresponding author: Email: zhwang@ asu.edu; Tel: +1-480-727-2933; Fax: +1-480-965-0557 


\section{Abstract}

2 Urban development is characterized by drastic land use and land cover changes, rooted in urban

3 population growth, with concomitant anthropogenic stressors contributing to global climate

4 changes. While there is extensive literature on predicting future urban climate using

5 biogeophysical models, the link between emergent meteorological patterns and the population

6 dynamics remains weak. In this study, we propose an operative model for predicting regional

7 urban warming using a population growth model based on different scaling laws. We choose the

8 annual average air temperature as the climatic indicator and relate the historical temperature data

9 to demographic data for 10 megapolitan areas in the United States. Linear regression analysis

10 shows that the coefficients of determination range from 0.164 to 0.563 for the study areas,

11 indicating a significant correlation between urban population and climate dynamics. Specifically,

12 the Phoenix Metropolitan Area is selected as a testbed where the regression is applied for

13 correlating the population growth and trend of urban warming. Based on the predicted population

14 dynamics, the annual average temperature in Phoenix is projected to increase by $0.65-3.40{ }^{\circ} \mathrm{C}$

15 in the next 35 years.

17 Keywords: Climate change; Population model; Sustainability; Urban expansion 


\section{Introduction}

From 1850 to 2005, the global emission of greenhouse gases (GHGs), such as carbon

21 dioxide $\left(\mathrm{CO}_{2}\right)$ and methane, have been continuously increasing at an unprecedented rate, mainly

22 due to anthropogenic emissions, with major contributors including fossil fuel combustion (Le

23 Quéré et al., 2009), urbanization (Kalnay \& Cai, 2003), and land use and land cover (LULC)

24 changes (Feddema et al., 2005). For example, Le Quéré et al. (2009) demonstrated that the total

$25 \mathrm{CO}_{2}$ emission from urbanization and fossil fuel burning amounted to $9.9 \pm 0.9 \mathrm{Pg} \mathrm{C} \mathrm{yr}^{-1}$ in 2008 .

26 On the other hand, fossil fuel combustion, urbanization, and LULC changes contribute

27 significantly to temperature increase in urban areas, though it is hard to separate the effects of

28 urbanization and LULC changes on temperature increase (Kalnay \& Cai, 2003). As a

29 consequence, the global average surface temperature has increased by around $0.76{ }^{\circ} \mathrm{C}$ in this

30 period (IPCC, 2007). The warming trend is projected to continue, e.g. Davis et al. (2010)

31 predicted an increase of $1.3{ }^{\circ} \mathrm{C}$ of global mean temperature in 2060 as compared to the

32 pre-industrial era, due to $\mathrm{CO}_{2}$ emissions from fossil fuel combustion.

The population growth, concomitant with the global economic development, is an

34 intrinsic driver of all the aforementioned contributors to climate changes (Rosa, York, \& Dietz,

35 2004), which in turn prompts urban development via, e.g. technological innovations and reduced

36 footprints of resource allocation per capita. In 1900, only about $40 \%$ of the total population was

37 an urban resident in the U.S.; this percentage was over 75\% in 1990 (Henderson, 2002). Urban

38 growth and the associated infrastructure development/retrofit, inevitably, lead to adverse 
39 environmental issues, such as the urban heat island (UHI) (Oke, 1973), air pollution (Mage et al.,

40 1996), and human health issues (Patz, Campbell-Lendrum, Holloway, \& Foley, 2005), to name a

41 few. For example, the anthropogenic heat, generated from human metabolism, industries,

42 buildings, vehicles, etc., is one of the main contributors to urban warming (Sailor, 2011). Sailor

43 (2011) reported that the metabolic heat during daytime in U.S. cities is around $1 \mathrm{~W} \mathrm{~m}^{-2}$. In

44 industrial sectors, sensible heat is the main form of anthropogenic heat as generated by energy

45 consumptions; accurate estimate of its magnitude is imperative but lacking (Sailor, 2011). Heiple

46 and Sailor (2008) pointed out that energy consumptions inside buildings (mainly induced by the

47 heating, ventilation and air conditioning systems) result in significant anthropogenic heat

48 emissions. Additional contribution to the anthropogenic heat (and moisture) arises from vehicular

49 emission as a result of fossil fuel combustion.

As a developed country, the U.S. has several cities of dense population, with more than

$5161 \%$ of the Nation's population distributed over 23 megapolitan areas (Nelson \& Lang, 2011).

52 Karl et al. (1988) quantified the correlation between increasing population and temperature

53 among a network of 1219 stations in the U.S. from 1901 to 1981 . The effect of urbanization on

54 temperature has been detected even in small towns (population < 10000). Balling and Idso (1989)

55 also demonstrated urban warming is associated with population growth based on historical

56 temperature data in U.S. from 1920 to 1984. The trend is expected to continue with further

57 urbanization and population concentration, which calls for a predictive demographic model

58 (Bettencourt, Lobo, Helbing, Kühnert, \& West, 2007), especially for cities, in order to devise an 
59 operative tool for future urban climate projections.

60 For predicting future climate changes, the existing studies usually adopt biogeophysical

61 models in conjunction with statistical techniques, such as the Coupled Model Inter-comparison

62 Project (CMIP) (Oleson, 2012) with statistical or dynamical downscaling to study areas of

63 interest. However, applications of such global models or reanalysis data products require

64 expensive computational resources and in-depth understandings of the underlying dynamics of

65 the integrated earth-atmosphere system. Instead, this study seeks an alternative way to

66 circumvent these limitations for quantifying climate changes, specifically in densely populated

67 regions. Our far-reaching goal is to develop a novel approach for predicting trends of urban

68 warming by quantitatively linking the population-climate dynamics in metropolitans. Towards

69 this end, we selected 10 megapolitan areas in the continental U.S. to statistically correlate the

70 historical annual average air temperature and the population size in each region. In addition, the

71 Phoenix Metropolitan Area (PMA) was chosen as a testbed for detailed statistical analysis. After

72 validation against the historical record, the proposed model is applied for future population

73 projection, upon which the future trend of urban temperature is then forecasted.

\section{2. Methods}

$76 \quad 2.1$ Urban population model

The population growth is an intrinsic measure of urbanization, which blends all the

78 dynamic indicators associated with urban development such as infrastructure development, 
79 LULC changes, anthropogenic emissions, etc. Define $N(t)$ as the population in a specific urban

80 region at time $t$, the material resources at that time as $Y(t)$, the co-evolution of the available

81 material resources and the sustained total population can be linked using a generic exponential

82 function (Bettencourt et al., 2007):

$$
Y(t)=Y_{0} N(t)^{\beta},
$$

84 where $Y_{0}$ is the initial resource, and $\beta$ is a parameter accounting for generic dynamic contributors

85 in a community (a generic one, be it a human society or a biological group) development

86 contributing to the population growth/decay. For example, a $\beta$ value smaller than unity signifies

87 the constraint of infrastructure and transportation materials/quantities in the built environment

88 (Bettencourt et al., 2007). While the urban infrastructure, e.g. gasoline stations and length of

89 electrical cables, is necessary in terms of urban development, its limitations of development or

90 availability inevitably impose restrictions on continuous population increase. This is analogous

91 to a biological community; e.g. for bees, when the food supply is ample, the available space in a

92 beehive will ultimately set an upper limit of the bee population.

93 The consumption of the total material resources can be subdivided into two categories, i.e.

94 for population maintenance and growth, respectively, which yields:

95

$$
Y(t)=R_{m} N(t)+E \frac{d N}{d t},
$$

96 where $R_{m}$ is the average amount of resource for individual maintenance per unit time per capita,

97 with the subscript $m$ denotes "model", and $E$ is the average amount of resource required for 98 population growth per capita. 
100 employment, household electrical and water consumptions), leading to an exponential growth

101 pattern. The exponential growth is well-known as a paradigmatic "free" growth mode for a

102 generic biological community (e.g., grey seals (Bowen, McMillan, \& Mohn, 2003)) or human

103 societies (e.g., Kampala, Uganda (Vermeiren, Van Rompaey, Loopmans, Serwajja, \& Mukwaya,

104 2012)), subject to no pressing external stress. Integrating Eq. (2) leads to:

105

$$
N(t)=N_{0} e^{\left(Y_{0}-R_{m}\right) t / E}
$$

106 Note that for the exponent here, $\left(Y_{0}-R_{m}\right) / E$ is time-independent. When $\beta>1$, population growth

107 is driven by wealth, information and resources creation (e.g., inventors, wages, GDP, etc.). Note

108 that in this case, there will be an initial capacity $\left(R_{m} / Y_{0}\right)^{1 /(\beta-1)}$. If the initial population $N_{0}$ is

109 greater than this capacity there exists a threshold time $t_{c}$ for population growth, after which

110 population will collapse:

$$
t_{c}=-\frac{E}{(\beta-1) R_{m}} \ln \left[1-\frac{R_{m}}{Y_{0}} N^{1-\beta}(0)\right] \approx\left[\frac{E}{(\beta-1) R_{m}}\right] \frac{1}{N^{\beta-1}(0)} .
$$

112 When $\beta<1$, the growth curve should be sigmoidal with a carrying capacity $\left(Y_{0} / R_{m}\right)^{1 /(1-\beta)}$, and

113 the growth is related to optimal and efficient urban development (e.g. infrastructure and 114 transportation materials or quantities).

115 For simplicity, $R_{m}$ and $E$ are assumed to be constants and independent of $N$, following the 116 treatment in previous studies (Lane, Pumain, van der Leeuw, \& West, 2009; West, Brown, \& 117 Enquist, 2001). Population growth during a few decades can be divided into several successive 
118 cycles or time periods (stages) (Bettencourt et al., 2007). In a single stage, the initial capacity

119 serves as a threshold for population growth with scaling $\beta>1$, while the carrying capacity serves

120 as the constraint for $\beta<1$. Hence, we prescribe $Y_{0}$ as a constant with a fixed $\beta$ value every year

121 in a single stage. The ratio $E / R_{m}$ is interpreted as the necessary average time for a human from

122 birth to productive maturity, given by $E / R_{m} \approx \tau \times 20$ years (Bettencourt et al., 2007), where $\tau=1$

123 year ${ }^{-1}$. Re-arrange Eq. (2), we have:

124

$$
N(t)=\left[\frac{Y_{0}^{*}}{R_{m}{ }^{*}}+\left[N^{1-\beta}(0)-\frac{Y_{0}^{*}}{R_{m}^{*}}\right] \exp \left[-\frac{R_{m}^{*}}{E^{*}}(1-\beta) t\right]\right]^{\frac{1}{1-\beta}} .
$$

125

\subsection{Population-temperature regression}

Here we use a statistical regression model to correlate the historical climate data to

128 demographic data:

$$
y=\beta_{0}+\beta_{1} x+\varepsilon,
$$

130 where $y$ is the response function, $x$ is the regressor, $\beta_{0}$ and $\beta_{1}$ are the coefficients of linear

131 regression, and $\varepsilon$ is a random error term. In order to estimate the regression coefficients in Eq. (6),

132 we adopted the least-squares estimation:

$$
\hat{y}=\hat{\beta}_{0}+\hat{\beta}_{1} x
$$

134 where the circumflex denotes estimated value (estimator), and the regression coefficients in Eq.

135 (6) are replaced by the least-squares estimators here. 


\section{Statistical Analysis in 10 U.S. Megapolitans}

140 climatic change and population growth in cities, we collected data from 10 major megapolitan

141 areas in the U.S. (Nelson \& Lang, 2011). The demographic data from 1969 to 2014 were

142 retrieved from the U.S. Department of Commerce, Bureau of Economic Analysis (BEA) (Brown

143 \& Wardwell, 1980; U.S. BEA, 2014). General geographic information, in particular, main cities,

144 states, and the number of counties located within or overlapping the geographical boundaries of

145 these megapolitan areas are summarized in Table 1.

147 Table 1. General geographic information of the 10 selected major megapolitan areas in the U.S.

\begin{tabular}{|c|c|c|c|}
\hline Megapolitan areas & Main cities & $\begin{array}{l}\text { Number of } \\
\text { counties }\end{array}$ & $\begin{array}{l}\text { State and counties inside } \\
\text { megapolitan areas }\end{array}$ \\
\hline Sun Corridor & Phoenix, Tucson & 4 & $\mathrm{AZ}$ \\
\hline Cascadia & Seattle, Portland, Vancouver & 24 & OR, WA and Canada \\
\hline Florida & Tampa, Miami, Orlando, Jacksonville & 25 & FL \\
\hline Front Range & $\begin{array}{l}\text { Albuquerque, Santa Fe, Colorado Springs, } \\
\text { Denver }\end{array}$ & 15 & $\mathrm{CO}, \mathrm{NM}$ \\
\hline Great Lakes & $\begin{array}{l}\text { Chicago, Detroit, Pittsburgh, Columbus, } \\
\text { Cleveland, Minneapolis, St. Louis, Indianapolis }\end{array}$ & 169 & $\begin{array}{l}\text { IL, IN, MI, MN, OH, PA, } \\
\text { WI, Canada }\end{array}$ \\
\hline Northeast & $\begin{array}{l}\text { New York, Philadelphia, Washington D.C., } \\
\text { Boston, Baltimore }\end{array}$ & 165 & $\begin{array}{l}\text { CT, DC, DE, MA, MD, NY, } \\
\text { NJ, PA, VA, WV, NH, RI }\end{array}$ \\
\hline $\begin{array}{l}\text { Northern } \\
\text { California (CA) }\end{array}$ & $\begin{array}{l}\text { Oakland, Reno, Sacramento, San Jose, San } \\
\text { Francisco }\end{array}$ & 27 & $\mathrm{CA}, \mathrm{NV}$ \\
\hline Piedmont Atlantic & $\begin{array}{l}\text { Atlanta, Birmingham, Raleigh-Durham, } \\
\text { Charlotte }\end{array}$ & 121 & $\mathrm{NC}, \mathrm{SC}, \mathrm{GA}, \mathrm{AL}$ \\
\hline $\begin{array}{l}\text { Southern } \\
\text { California (CA) }\end{array}$ & $\begin{array}{l}\text { Los Angeles, San Diego, Anaheim, Long } \\
\text { Beach, Las Vegas }\end{array}$ & 12 & $\mathrm{CA}, \mathrm{NV}$ and Mexico \\
\hline
\end{tabular}




\begin{tabular}{llllll}
\hline Texas Triangle & $\begin{array}{l}\text { Austin, Dallas/Fort } \\
\text { Antonio }\end{array}$ & Worth, Houston, San 67 & TX \\
\hline
\end{tabular}

150 collected at the county level, i.e., the total population of a megapolitan area is the sum of

151 population from individual counties. For example, population in Great Lakes is the sum of

152 population from 169 counties in this area (see Table 1). During the past 46 years $(1969-2014)$,

153 the population in all the 10 megapolitans has experienced continuous increases (Figure 1), except

154 for the Great Lakes where fluctuations of population were observed around 1979 - 1986. Though

155 not the largest megapolitan area in the U.S., the Northeast region is the most densely populated

156 megapolitan with the largest population size as well as significant growth rate. Among these

157 regions, Arizona's Sun Corridor has seen the most rapid rate of urbanization with a population

158 growth of more than $309 \%$ during the past five decades. The rate of population growth is lowest

159 in Great Lakes and Northeast, which is partly due to the high level of urbanization preexisted in

160 these areas prior to 1969. 

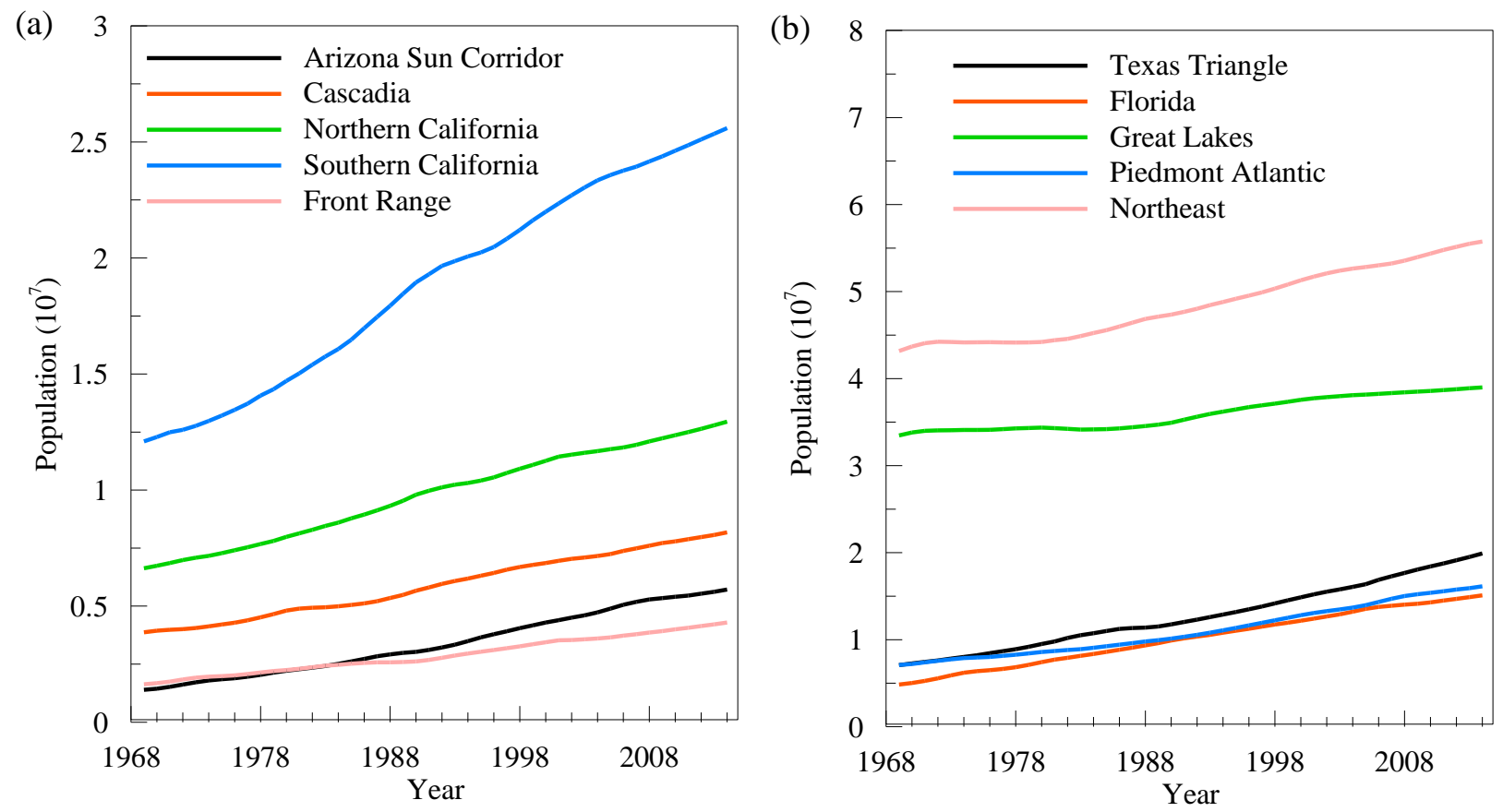

163 Figure 1. Population growth in the selected megapolitan areas in (a) Sun Corridor, Cascadia,

164 Northern California, Southern California, and Front Range, and (b) Texas Triangle, Florida,

165 Great Lakes, Piedmont Atlantic, and Northeast during 1969 - 2014.

The temperature data were downloaded from the National Environmental Satellite, Data,

168 and Information Service (NESDIS) of the National Oceanic and Atmospheric Administration

169 (NOAA) (NOAA NESDIS, 2016). We averaged the archived near-surface $(5 \pm 1 \mathrm{ft})$ air

170 temperature of all climate divisions in each megapolitan area to obtain the annual average

171 temperature (AAT) of an individual megapolitan. All the 10 selected regions exhibit a trend of

172 urban warming with some fluctuations (Figure 2) under the background of global climate

173 changes accelerated in the past half century (Murray \& Colle, 2011). Florida, Sun Corridor and

174 Texas Triangle are the three warmest megapolitan areas in the U.S. during the past 46 years, 

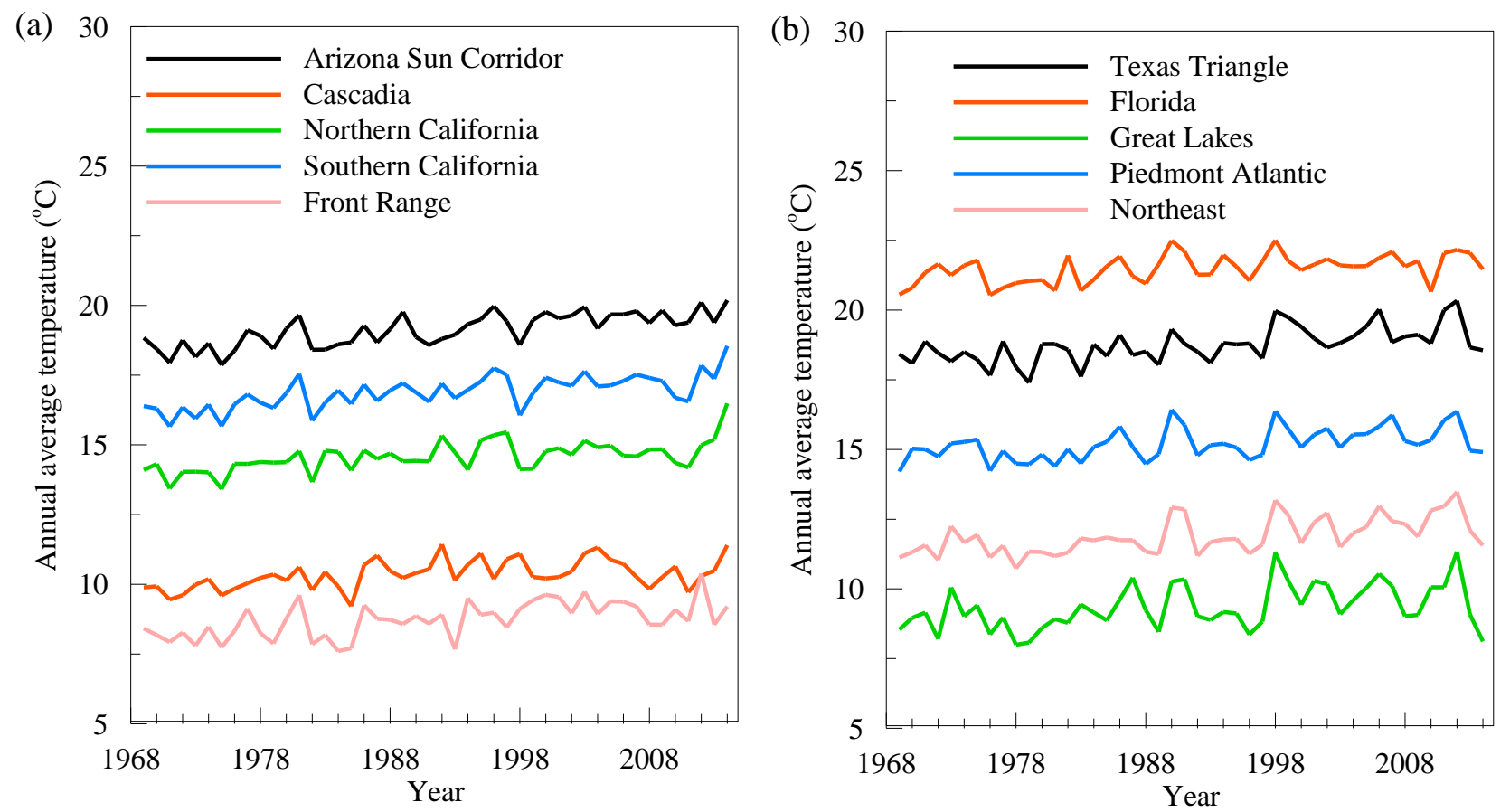

179 Figure 2. Same as Figure 1 but for AAT. To quantify the correlation between the population growth and urban warming, we

183 The coefficient of determination $R^{2}$ is used to quantify the correlation level. It is clear from

184 Figure 3 that the AAT is significantly correlated with population in most of these megapolitan

185 areas. The statistical correlation is found to be most significant in Arizona's Sun Corridor, with a $R^{2}$ value of 0.563 . The variance is expected as that the AAT data is obtained from the divisional

187 level with areal averaging and embedded background changes without discriminating to detailed 
188 land use land cover classification, whereas the population growth is obtained at county level.

189 Relation between AAT and population in Great Lakes during the past 46 years indicates large

190 data scatter relative to Arizona's Sun Corridor. Two megapolitan areas in California show similar

191 high $R^{2}$ values to Arizona's Sun Corridor, especially Southern California $\left(R^{2}=0.468\right)$, revealing

192 similar temperature-population relation in this geographical region (Arizona and California),

193 while the correlation Great Lakes is slightly at variance with the general trend with a relatively

194 low $R^{2}$ value of 0.164 .

195 

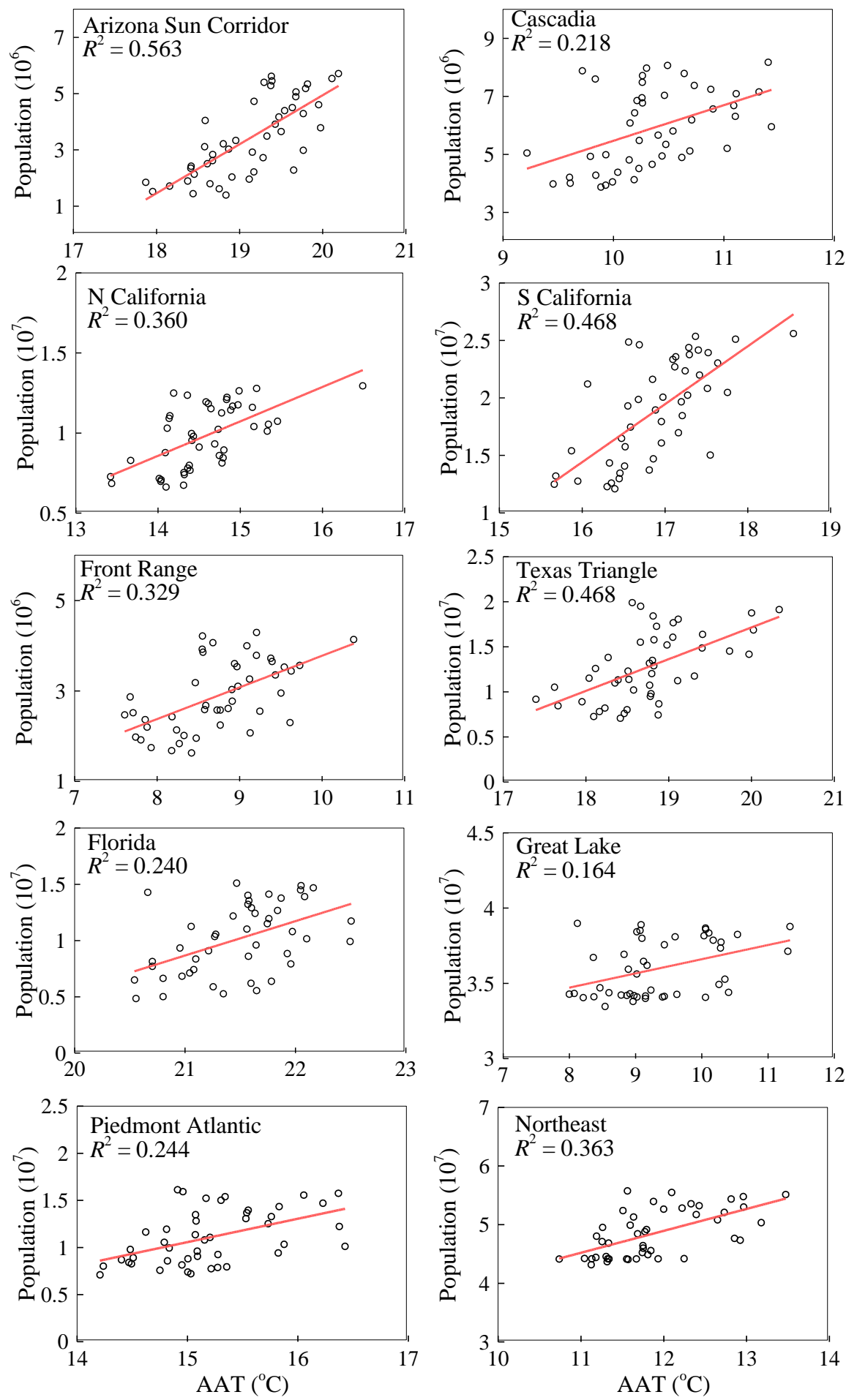

197 Figure 3. Statistical correlation between the AAT and population in the 10 megapolitan areas in

198 the period of 1969 - 2014; scatter points are the historical observations, and red line is the linear

199 regression. 


\section{4. Case Study in the Phoenix Metropolitan Area}

\section{$201 \quad 4.1$ The study area}

202 In this study, we chose the Phoenix Metropolitan Area (PMA) as out testbed, partly

203 informed by the strongest population-temperature correlation in Sun Corridor. As the largest

204 metropolitan area in this region, the PMA is a desert metropolitan area of about $37000 \mathrm{~km}^{2}$ and

205 has been expanding for more than 60 years since 1950 (Brazel et al., 2007) (Figure 4). During

206 this period, extensive LULC changes have taken place, converting agricultural and natural

207 landscapes to the built environment. The urban expansion of the PMA was one of the highest

208 among 100 largest metropolitan areas from 1980 to 2010 (Frey, 2012). Rapid urbanization in this

209 region results in not only a population growth, but also temperature increases and a severe UHI

210 (Hedquist \& Brazel, 2014). For example, Brazel et al. (2007) reported an increase of 2 to $4{ }^{\circ} \mathrm{C}$ in

211 mean minimum temperature of June in this region due to urbanization between 1990 and 2004. 


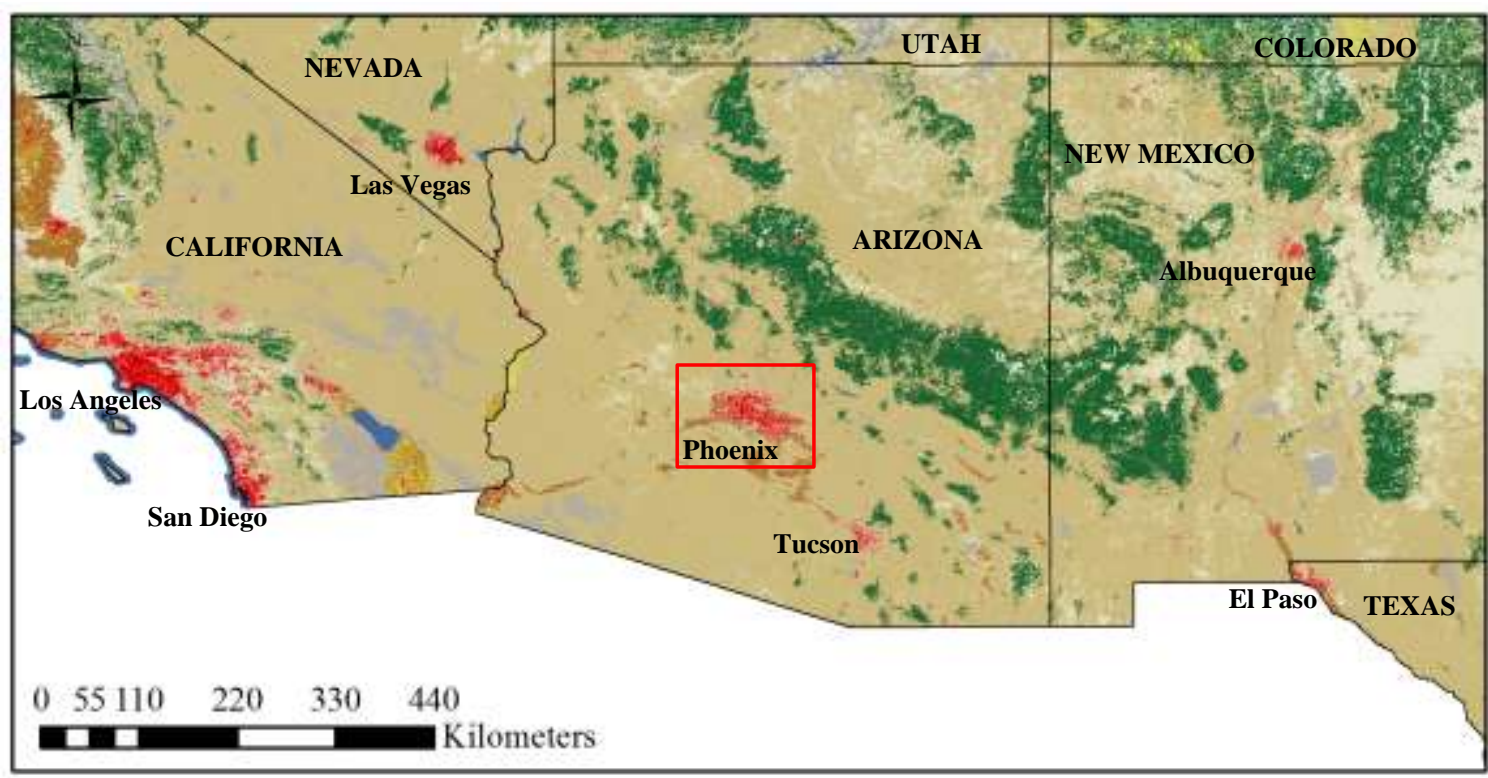

- Weather Stations

NLCD Land Cover Classification Legend 11 Open Water

12 Perennial Ice/ Snow

21 Developed, Open Space

22 Developed, Low Intensity

23 Developed, Medium intensity

24 Developed, High Intensity

31 Barren Land (Rock/Sand/Clay)

41 Deciduous Forest

42 Evergreen Forest

43 Mixed Forest

51 Dwart Scrub*

62 Shrub/Scrub

71 Grassland/Herbaceous

72 Sedge/Herbaceous*

73 Lichens*

74 Moss*

81 Pasture/Hay

82 Cultivated Crops

30 Woody Wetlands

95 Emergent Herbaceous Wetlands

- Alaska onty

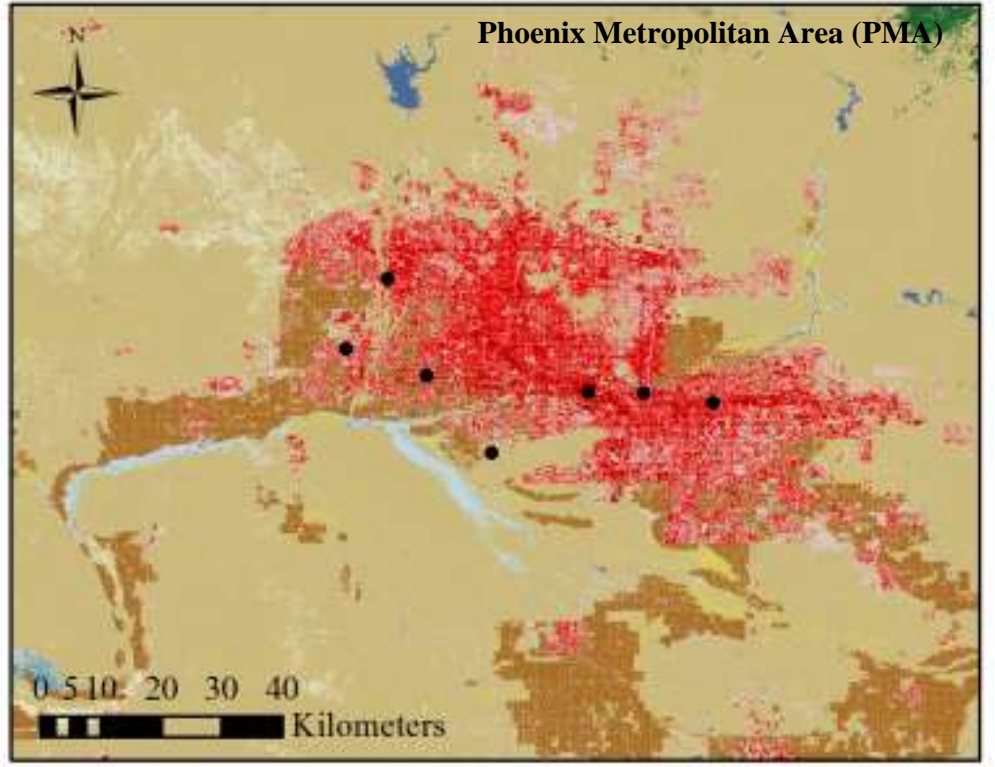

213 Figure 4. Geographical location of the study area with national land cover (NLCD2011) (a)

214 overlaid in the state of Arizona, and (b) PMA and weather stations 
218 from BEA (2014) were used in this analysis. Population growth rates of the low, medium and

219 high series (2015-2050) were retrieved from the Population Projections of the Arizona 220 Department of Administration - Office of Employment and Population Statistics (ADOA-EPS)

221 (2015). In addition, 7 NOAA land-based weather stations (NOAA NESDIS, 2016) were selected 222 for the estimate of historical AAT data (1969 - 2014) (Figure 4b). Results for the correlation 223 between the historical AAT and population data in the PMA using both the linear and exponential 224 regression are shown in Figure 5.

225
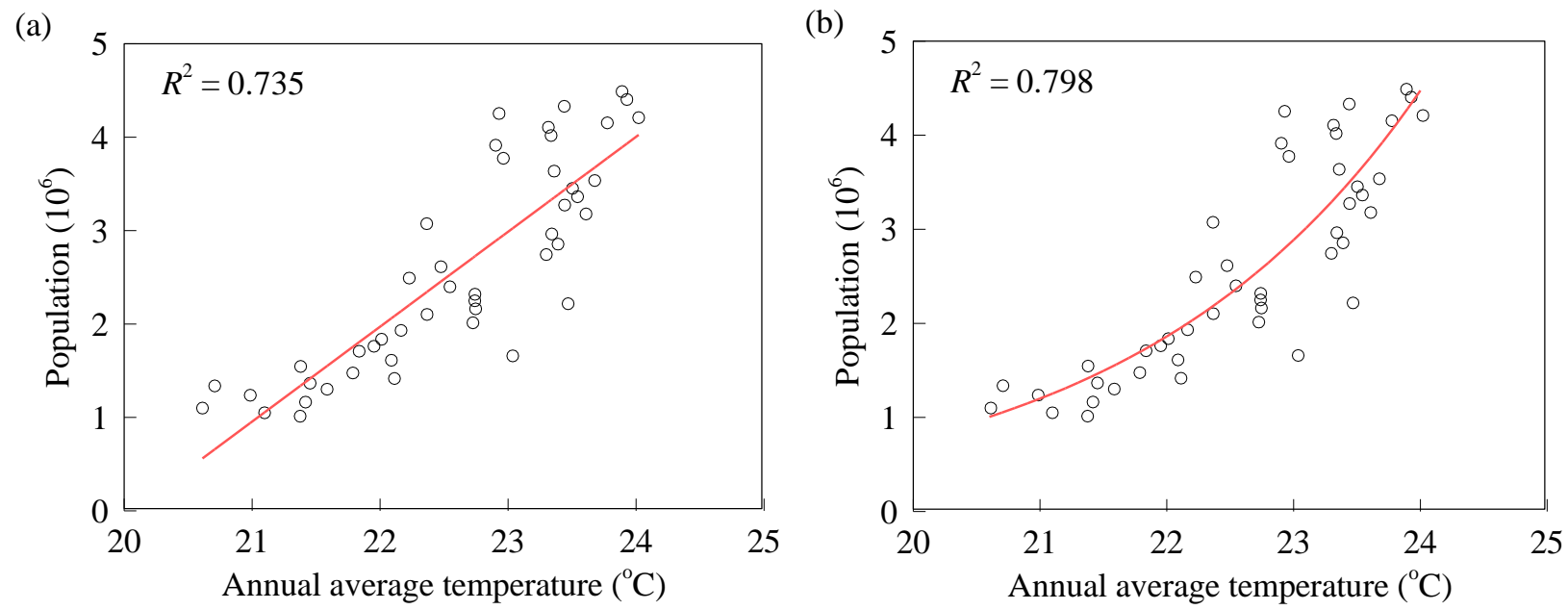

226

227 Figure 5. Correlation between the AAT and population in the PMA using the (a) linear and (b)

228 exponential regression.

229

The temperature-population relation is determined statistically as

$$
T=0.0723 N+20.7344,
$$

232 using the linear regression, where $T$ is the temperature in Celsius, and $N$ is the population in $10^{5}$; 
233 and

$$
T=1.8201 \ln N+16.8590 \text {. }
$$

235 using the exponential regression. Significant coefficients of statistical correlation were found

236 between the populating and urban warming in both the linear $\left(R^{2}=0.735\right)$ and exponential $\left(R^{2}=\right.$ 237 0.798) regression (at the confidence level of 95\%).

\section{$239 \quad 4.3$ Simulating the historical population growth}

240 In this section, the future population growth in the PMA is projected using the prognostic

241 population model (see Section 2.1 for details), where various growth modes represent different

242 underlying driving forces for population increases, including technological, socio-economic, and

243 political drivers. Relative population growth rate of the PMA over time revealed the existence of

244 accelerating and decelerating stages (Figure 6), similar to those of New York City (Bettencourt et

245 al., 2007). However, due to different urbanization levels in these two regions, the successive

246 shorter stages of super-exponential growth in New York City are not observed in the PMA. The

247 reaccelerated process during a different period was strongly modulated by new emerging urban

248 areas in the PMA, as shown in Table 2. To test the applicability of the proposed population

249 model in simulating different population growth modes, we divide years 1969-2014 into seven 250 different stages, with the key parameters listed in Table 3. 


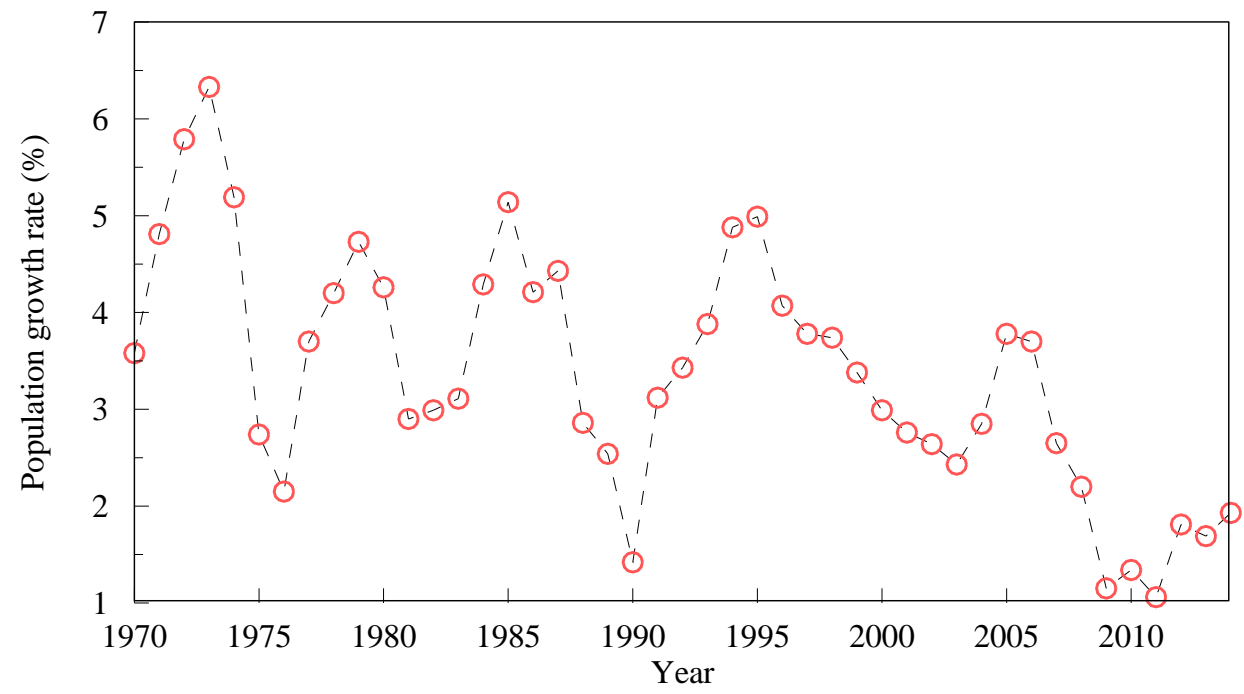

253 Figure 6. Historic record of population growth rate in PMA from 1969 to 2014.

255 Table 2. Emerging cities during different population growth stages in the PMA.

Years Emerging cities and population growth facts

1969-1976 Available data since 1969(U.S. BEA, 2014).

The recession during 1969 - 1970 resulted in a population $\operatorname{spurt}(\operatorname{Rex}, 2000)$.

1977-1981 Glendale, Tempe, Mesa experienced their highest population growth rates(U.S. BEA, 2014).

1982-1990 East Valley (Mesa, Chandler, Tempe, and Gilbert) emerged around 1982(Ripley, 2011) http://www.eastvalleytribune.com/guide/article_ac67b832-fa97-11e0-b1eb-001cc4c03286.html). Peoria went through its highest population growth rate (over 300\%)(U.S. BEA, 2014).

1991-2003 Chandler experienced an exponential growth (early 1990s), meanwhile Surprise and Avondale went through their highest population growth rates (over $300 \%$ and over 100\%, respectively) (U.S. BEA, 2014).

2004-2011 Goodyear, Buckeye experienced the highest population growth rate (over 200\% and over 600\%, respectively). Surprise and Avondale continued to expand (population increased over $300 \%$ and over $100 \%$, respectively). Population in El Mirage increased over 300\%, and the increase in Queen Creek was over 500\%(U.S. BEA, 2014).

2012-2014 Population growth has the same trend as that of the state of Arizona (ADOA-EPS, 2015), and this may be related to economy recovery. 
257 Table 3. Calibrated parameters in the proposed model for different stages of population growth 258 in PMA.

\begin{tabular}{llllllll}
\hline Period index & Stage & $N_{0}\left(1 \times 10^{5}\right)$ & $\beta$ & Error $<=$ & $R_{m}{ }^{*}$ & $E^{*}$ & $R^{2}$ \\
\hline (a) & $1969-1973$ & 10.134 & 1.211 & $1.5 \% \mathrm{~N}(\mathrm{t})_{\text {real }}$ & 0.50 & 1.00 & 0.992 \\
(b) & $1974-1979$ & 13.020 & 1.300 & $1.5 \% \mathrm{~N}(\mathrm{t})_{\text {real }}$ & 0.50 & 1.00 & 0.985 \\
(c) & $1980-1985$ & 16.122 & 1.282 & $1.5 \% \mathrm{~N}(\mathrm{t})_{\text {real }}$ & 0.50 & 1.00 & 0.989 \\
(d) & $1986-1990$ & 20.133 & 0.853 & $0.5 \% \mathrm{~N}(\mathrm{t})_{\text {real }}$ & 0.50 & 1.00 & 0.999 \\
(e) & $1991-2003$ & 23.192 & 0.848 & $1.5 \% \mathrm{~N}(\mathrm{t})_{\text {real }}$ & 0.50 & 1.00 & 0.998 \\
(f) & $2004-2011$ & 36.373 & 0.877 & $1.5 \% \mathrm{~N}(\mathrm{t})_{\text {real }}$ & 0.50 & 1.00 & 0.995 \\
(g) & $2012-2014$ & 43.310 & 1.129 & $0.1 \% \mathrm{~N}(\mathrm{t})_{\text {real }}$ & 0.50 & 1.00 & 0.999 \\
\hline
\end{tabular}

Figure 7 illustrates the capacity of the population growth model in predicting population

262 in different temporal periods as well as growth modes. Note that a superlinear $(\beta>1)$ relation is

263 found for four periods (Figure 7a-c\&g) in the PMA, suggesting the information and resources

264 creation, such as patents and financial services, as the main driving force during these periods.

265 Taking innovation for example, metropolitan areas are often more appealing to inventors and

266 industries (Batty, 2008; Feldman \& Audretsch, 1999). The larger $\beta$ value found in the PMA

267 suggests rapid population growth rate associated with strong driving force in the area during the 268 above superlinear growing periods. 

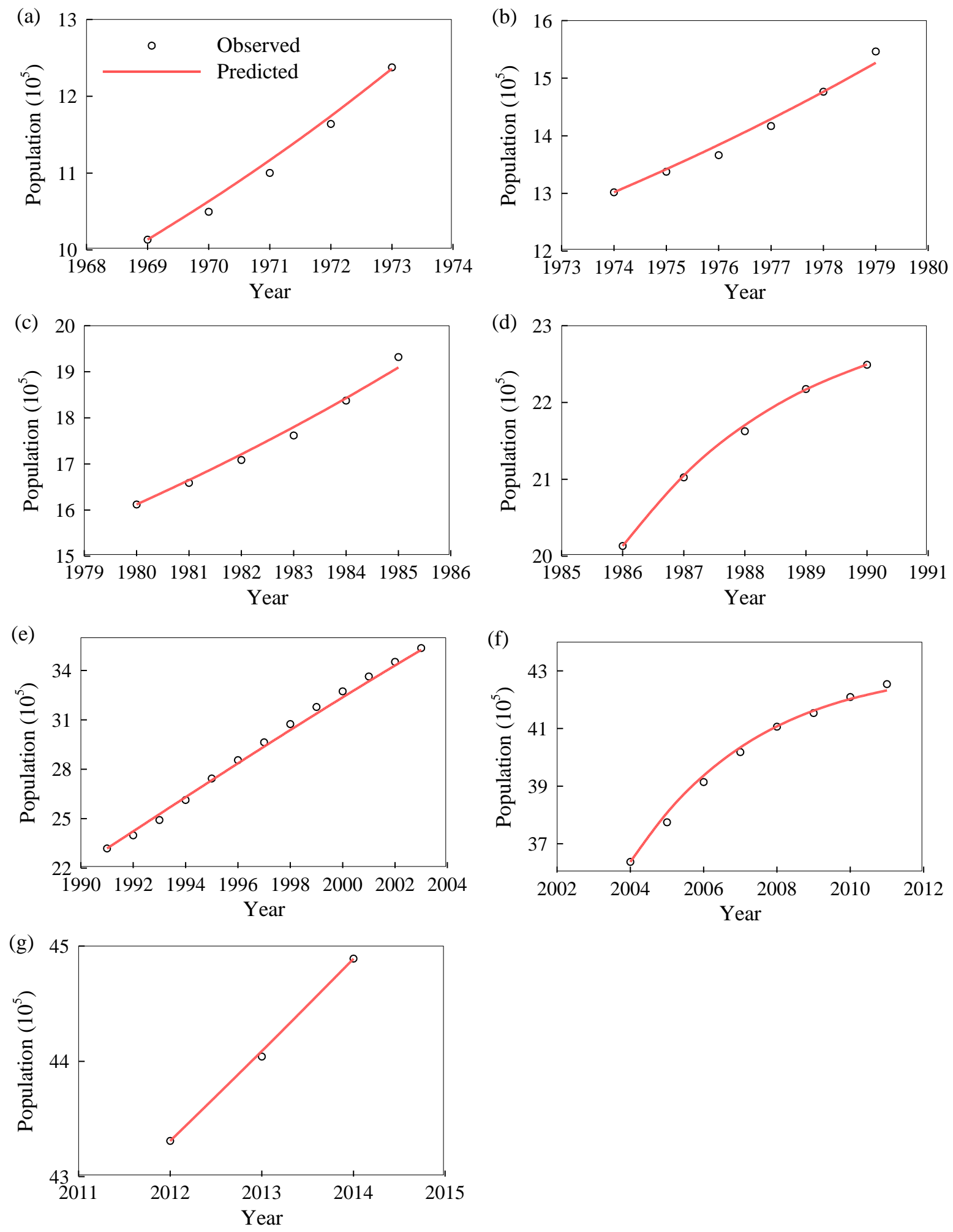

Figure 7. Comparison between predicted and observed historical population in the PMA;

272 numbering of subplots follows the index in Table 3. 
275 calibrated parameters of the last stage $(2012$ - 2014), we predict the future population in the

276 PMA up to 2050, with results shown in Figure 8. Here we denote the two theoretical population

277 models derived from Bettencourt et al. (2007) as: (a) the free growth model based on Eq. (5) with

$278 \beta=1.0$, and (b) the scaling model with $\beta>1$ or $\beta<1$ for super-exponential or sub-exponential

279 growth respectively, each constrained by a different set of socioeconomic conditions. In addition

280 to the theoretical predictions, the low, medium, and high series population growth rates projected

281 empirically by the ADOA-EPS (2015) are also presented in Figure 8. A more rapid rate of

282 population increase predicted by the scaling model $(\beta=1.129)$ is observed as compared to that of

283 the free growth model $(\beta=1.0)$. Meanwhile, both theoretical models predict larger population

284 sizes than the empirical ADOA-EPS population projections in 2050. At the end of 2050, using

285 the medium series of ADOA-EPS projection as the reference, the deviation of the population

286 estimated by the scaling model from the reference is $24.29 \%$, while the discrepancy of the free

287 growth model is $13.74 \%$. This discrepancy apparently suggests that the uncertainty of the

288 theoretical predictions of future population becomes larger as expected, where a local stationarity

289 is assumed in the population growth pattern and extended for a long period of time (in this case

29035 years from 2016 to 2050). 


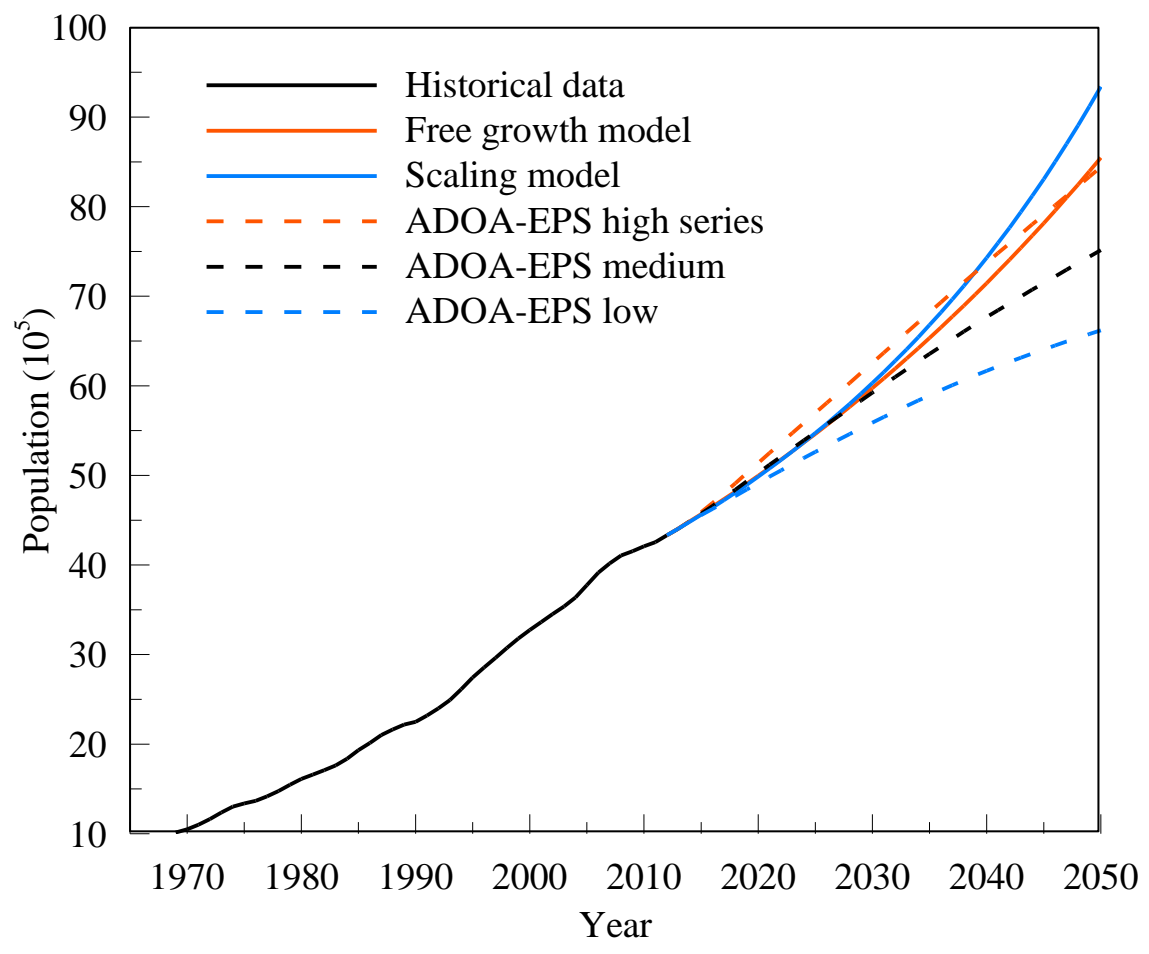

293 Figure 8. Projected future population in the PMA based on calibrated parameters in the most

294 recent period of $2012-2014$.

Both the free growth and the scaling models can predict the population size in the first 15

297 years (2016 - 2030) with reasonable accuracy when compared against projection of the medium

298 series, with an error less than 1\%. The theoretical models underestimate population size from

2992015 to 2025 , but with slight overestimates from 2025 to 2030 . A more significant deviation is

300 observed between predicted and projected population from 2030 to 2050, indicating that key

301 parameters for the theoretical population model should be updated periodically to represent

302 dynamic population growth mode in a city, especially for regions with rapidly varying

303 socioeconomic conditions (e.g. technology hubs). 
306 temperature if a feasible population growth model is given, where both the linear regression

307 (hereafter referred to as LR) in Eq. (8) and the exponential regression (hereafter ER) in Eq. (9)

308 are used in subsequent analysis. The predicted temperature trends in the PMA using the

309 population projections estimated from the free growth and scaling models, together with that

310 from the medium series projection by ADOA-EPS, are shown in Figure 9, based on LR and ER

311 population-temperature correlations respectively. It is clear that predicted future temperatures

312 using LR are higher than those using ER, due to the superlinear characteristic of ER (i.e., the

313 temperature increase rate slows down when population size is large, c.f. Figure 5b). All

314 projections of temperature increase are summarized in Table 4. The scaling model with LR

315 predicts the strongest warming from $24.1^{\circ} \mathrm{C}$ to $27.5{ }^{\circ} \mathrm{C}$ over the next 35 years, while the low

316 series population growth with ER yields the weakest warming from $23.8{ }^{\circ} \mathrm{C}$ to $24.5{ }^{\circ} \mathrm{C}$. In 2050 ,

317 the predicted temperature increase using LR relative to the reference temperature in 2016 ranges

318 from $1.44{ }^{\circ} \mathrm{C}$ to $3.40{ }^{\circ} \mathrm{C}$, while the increase using ER varies from $0.65{ }^{\circ} \mathrm{C}$ to $1.27{ }^{\circ} \mathrm{C}$ for different

319 population growths. 


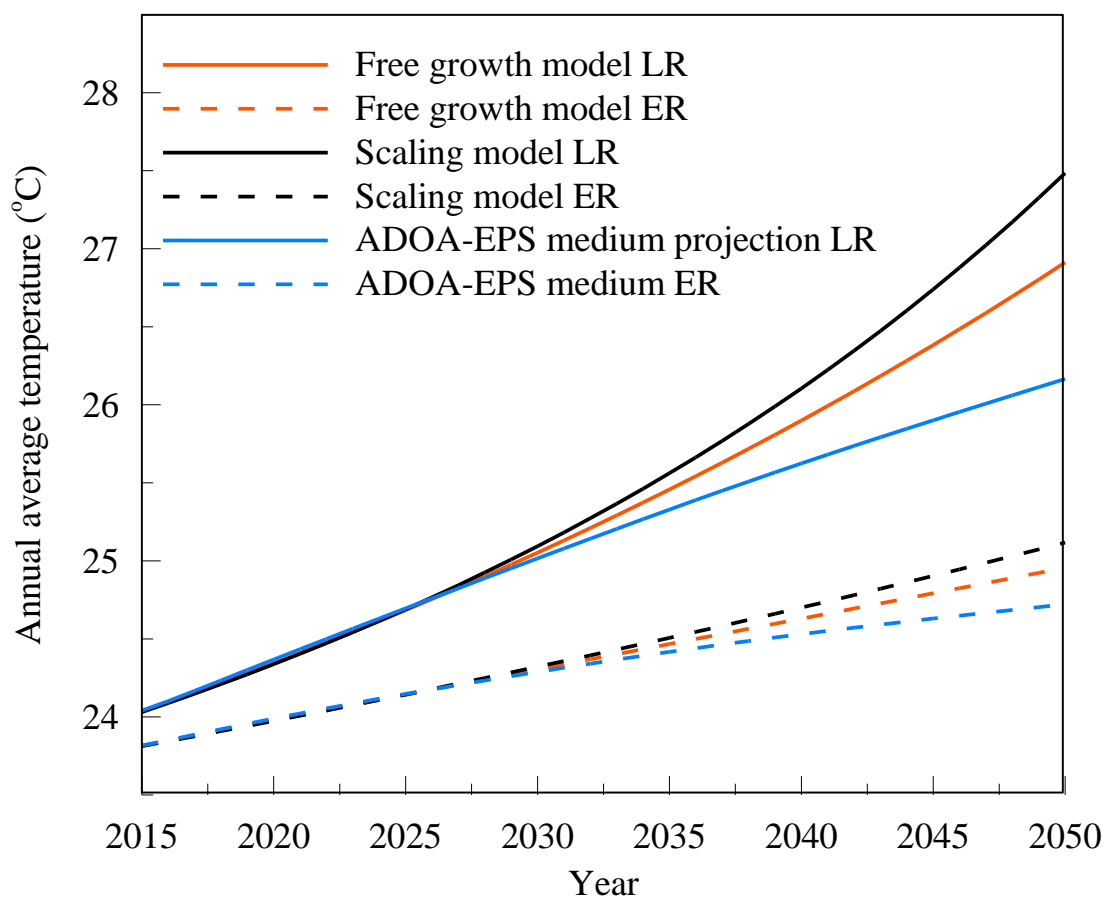

321

322 Figure 9. Predicted trends of future temperature changes in PMA using different population

323 projections.

324

325 Table 4. Summary of predicted temperature increase (in ${ }^{\circ} \mathrm{C}$ ) from the reference in 2016.

\begin{tabular}{lllllllllll}
\hline \multirow{2}{*}{ Year } & \multicolumn{9}{l}{ Linear regression (LR) } & \multicolumn{7}{c}{ Exponential regression (ER) } \\
\cline { 2 - 11 } & SM & FG & HS & MS & LS & SM & FG & HS & MS & LS \\
\hline 2016 & - & - & - & - & - & - & - & - & - & - \\
2020 & 0.25 & 0.25 & 0.32 & 0.27 & 0.21 & 0.13 & 0.13 & 0.17 & 0.14 & 0.11 \\
2025 & 0.60 & 0.59 & 0.73 & 0.59 & 0.46 & 0.30 & 0.29 & 0.35 & 0.30 & 0.23 \\
2030 & 1.01 & 0.96 & 1.13 & 0.91 & 0.70 & 0.48 & 0.46 & 0.52 & 0.44 & 0.34 \\
2035 & 1.47 & 1.36 & 1.54 & 1.23 & 0.92 & 0.66 & 0.62 & 0.68 & 0.57 & 0.44 \\
2040 & 2.02 & 1.80 & 1.94 & 1.52 & 1.11 & 0.86 & 0.78 & 0.82 & 0.68 & 0.52 \\
2045 & 2.65 & 2.29 & 2.33 & 1.80 & 1.29 & 1.06 & 0.94 & 0.95 & 0.78 & 0.59 \\
2050 & 3.40 & 2.82 & 2.71 & 2.06 & 1.44 & 1.27 & 1.11 & 1.07 & 0.87 & 0.65 \\
\hline
\end{tabular}

326 * SM: Scaling model; FG: Free growth model; HS: High series; MS: Medium series; LS: Low series 
In addition, we found that with LR relation, the predicted temperature increase by the

329 scaling model of population growth is close to that by the free growth model in the period 2016 -

3302035 , with a difference of only $0.1{ }^{\circ} \mathrm{C}$. Differences of the same magnitude are also observed with

331 ER projections from 2016 to 2050. Despite the difference in population growth modes (free

332 versus super- or sub-exponential), the predicted temperature in 2050 using the theoretical

333 population models is higher than that based on the empirical ADOA-EPS population projections,

334 as the latter takes into more socioeconomic constraints. Though the future PMA temperature

335 increase predicted by the ADOA-EPS is lower, considerable discrepancies still exist between

336 different series (vary from $0.42{ }^{\circ} \mathrm{C}$ to $1.27{ }^{\circ} \mathrm{C}$ ), indicating uncertain warming effects with

337 different population growth modes. Note that the warming range results from assumed different

338 total fertility rates of all race groups in 2100 (ADOA-EPS, 2015), viz., ADOA-EPS projects total

339 fertility rates will converge to $1.4,1.9$, and 2.4 for low, medium, and high series in 2100 ,

340 respectively.

342 5. Concluding Remarks

343 This study proposed a novel method for predicting urban climate changes in terms of the

344 environmental temperature based on the population dynamics, without resorting to complex

345 climate models. Data from 10 major megapolitan areas in U.S. are analyzed for the period from

3461969 to 2014. Reasonable coefficients of determination ranging from 0.164 (Great Lakes) to

3470.563 (Arizona Sun Corridor) suggest that correlation between AAT and population is 
348 statistically significant in the selected megapolitan areas. Specifically, the PMA with strong

349 population-warming correlation was used as our testbed, and both linear and exponential

350 regressions are tested with historical as well as projected temperature and population dataset. The

351 proposed population growth models were validated using the historical demographic data from

3521969 to 2014 in the study area. Furthermore, the predicted future population growth from

353 2016-2050 using the proposed model is found in reasonable agreement with the estimates from

354 the ADOA-EPS for the first 15 years, with higher model uncertainty manifested in longterm

355 projections.

356 Large discrepancies and higher uncertainty in longterm population projections indicate

357 that periodical update of key model parameters is required for using urban population as a

358 dynamic measure of regional climate change. This is necessary due to the fact that the urban

359 development and/or redevelopment in individual metropolitan areas over a long period inevitably

360 involve different modes and socio-economic drivers of population growth. In addition, with

361 richer sets of demographic, socio-economic, and climatic data products and refined

362 spatio-temporal analysis, the accuracy of the operative model proposed in this study is expect to

363 be enhanced in predicting future urban climate. Comparisons against temperature outputs from

364 climate models, especially those downscaled from global and regional reanalysis dataset or

365 general circulation models, can further evaluate the performance of the proposed model, but

366 hitherto remain as an open challenge. Overall, the proposed model provides a new perspective

367 for urban climate research, and is expected to be informative to environmental sustainability 
368 studies in general.

369

370 Acknowledgement

371 This work is supported by the National Science Foundation (NSF) [grant numbers

372 CBET-1435881, CBET-1444758], and National Transportation Center (NTC) @ Maryland [grant

373 number DTRT13-G-UTC30].

374 


\section{References}

376

ADOA-EPS. (2015). Arizona Department of Administration - Office of Employment and Population Statistics (ADOA-EPS) Maricopa County 2015-2050 Projections. Retrieved July 8, 2016, from https://population.az.gov/population-projections

Balling, R., \& Idso, S. (1989). Historical temperature trends in the United States and the effect of urban population growth. Journal of Geophysical Research: Atmospheres, 94(D3), 3359-3363.

Batty, M. (2008). The size, scale, and shape of cities. Science, 319(5864), 769-771.

Bettencourt, L. M., Lobo, J., Helbing, D., Kühnert, C., \& West, G. B. (2007). Growth, innovation, scaling, and the pace of life in cities. Proceedings of the National Academy of Sciences, 104(17), 7301-7306.

Bowen, W. D., McMillan, J., \& Mohn, R. (2003). Sustained exponential population growth of grey seals at Sable Island, Nova Scotia. ICES Journal of Marine Science: Journal Du Conseil, 60(6), 1265-1274.

Brazel, A., Gober, P., Lee, S., Grossman-Clarke, S., Zehnder, J., Hedquist, B., \& Comparri, E. (2007). Determinants of changes in the regional urban heat island in metropolitan Phoenix (Arizona, USA) between 1990 and 2004. Climate Research, 33(2), 171-182.

Brown, D. L., \& Wardwell, J. M. (1980). New directions in urban-rural migration: the population turnaround in rural America. New York, USA: Academic Press, Inc.

Davis, S. J., Caldeira, K., \& Matthews, H. D. (2010). Future $\mathrm{CO}_{2}$ emissions and climate change 
from existing energy infrastructure. Science, 329(5997), 1330-1333.

Feddema, J. J., Oleson, K. W., Bonan, G. B., Mearns, L. O., Buja, L. E., Meehl, G. A., \& Washington, W. M. (2005). The importance of land-cover change in simulating future climates. Science, 310(5754), 1674-1678.

Feldman, M. P., \& Audretsch, D. B. (1999). Innovation in cities: Science-based diversity, specialization and localized competition. European Economic Review, 43(2), 409-429.

Frey, W. H. (2012). Population growth in metro America since 1980: putting the volatile 2000s in perspective. Washington DC, USA: The Brookings Institution.

Hedquist, B. C., \& Brazel, A. J. (2014). Seasonal variability of temperatures and outdoor human comfort in Phoenix, Arizona, USA. Building and Environment, 72, 377-388.

Heiple, S., \& Sailor, D. J. (2008). Using building energy simulation and geospatial modeling techniques to determine high resolution building sector energy consumption profiles. Energy and Buildings, 40(8), 1426-1436.

IPCC. (2007). Climate Change 2007: The Physical Science Basis. Contribution of Working Group I to the Fourth Assessment Report of the Intergovernmental Panel on Climate

413 Kalnay, E., \& Cai, M. (2003). Impact of urbanization and land-use change on climate. Nature, 414 423(6939), 528-531. 
415 Karl, T. R., Diaz, H. F., \& Kukla, G. (1988). Urbanization: Its detection and effect in the United 416 States climate record. Journal of Climate, 1(11), 1099-1123.

417 Lane, D., Pumain, D., van der Leeuw, S. E., \& West, G. (2009). Complexity perspectives in $418 \quad$ innovation and social change. New York, USA: Springer.

419 Le Quéré, C., Raupach, M. R., Canadell, J. G., Marland, G., Bopp, L., Ciais, P., ... Foster, P. 420 (2009). Trends in the sources and sinks of carbon dioxide. Nature Geoscience, 2(12), $831-836$.

422 Mage, D., Ozolins, G., Peterson, P., Webster, A., Orthofer, R., Vandeweerd, V., \& Gwynne, M. 423 (1996). Urban air pollution in megacities of the world. Atmospheric Environment, 30(5), $424 \quad 681-686$.

425 Murray, J. C., \& Colle, B. A. (2011). The spatial and temporal variability of convective storms 426 over the northeast United States during the warm season. Monthly Weather Review, 139(3), 992-1012.

428 Nelson, A. C., \& Lang, R. E. (2011). Megapolitan America: A New Vision for Understanding 429 America’s Metropolitan Geography. Chicago, USA: American Planning Association.

NOAA NESDIS. (2016). National Climatic Data Center (NCDC) Climate Data Online (CDO). Retrieved June 27, 2016, from http://www7.ncdc.noaa.gov/CDO/georegion

432 Oke, T. R. (1973). City size and the urban heat island. Atmospheric Environment, 7(8), 769-779.

433 Oleson, K. (2012). Contrasts between urban and rural climate in CCSM4 CMIP5 climate change scenarios. Journal of Climate, 25(5), 1390-1412. 
Patz, J. A., Campbell-Lendrum, D., Holloway, T., \& Foley, J. A. (2005). Impact of regional climate change on human health. Nature, 438(7066), 310-317.

Rex, T. R. (2000). Development of metropolitan Phoenix: Historical, current and future trends. Tempe, USA: Morrison Institute for Public Policy, Arizona State University.

Ripley, J. (2011). The East Valley: 30 years and counting. Retrieved July 14, 2016, from http://www.eastvalleytribune.com/guide/article_ac67b832-fa97-11e0-b1eb-001cc4c03286 .html

Rosa, E. A., York, R., \& Dietz, T. (2004). Tracking the anthropogenic drivers of ecological impacts. AMBIO: A Journal of the Human Environment, 33(8), 509-512.

444 Sailor, D. J. (2011). A review of methods for estimating anthropogenic heat and moisture emissions in the urban environment. International Journal of Climatology, 31(2), 189-199.

U.S. BEA. (2014). U.S. Bureau of Economic Analysis (BEA). Retrieved June 23, 2016, from http://www.bea.gov/iTable/iTable.cfm?reqid=70\&step=1\&isuri=1\&acrdn=2\#reqid=70\&st ep=1\&isuri $=1 \& 7003=900 \& 7022=11 \& 7023=7 \& 7001=711 \& 7002=2 \& 7090=70$

Vermeiren, K., Van Rompaey, A., Loopmans, M., Serwajja, E., \& Mukwaya, P. (2012). Urban 454 growth of Kampala, Uganda: Pattern analysis and scenario development. Landscape and Urban Planning, 106(2), 199-206.

West, G. B., Brown, J. H., \& Enquist, B. J. (2001). A general model for ontogenetic growth. Nature, 413(6856), 628-631. 